\title{
Drug Repurposing Patent Applications October-December 2020
}

\section{Hermann A.M. Mucke}

H.M. Pharma Consultancy, Wien, Austria.

A RUSSIAN PSYCHOSTIMULANT FOR FIBROTIC DISEASES International Patent Application WO/2020/191503, Methods and Uses of Bromantane and Derivatives Thereof for Treating Lung Disease, Fatty Liver Disease, and Kidney Disorders (Inventor: Williams M; applicant: Algernon Pharmaceuticals, Inc., Vancouver, Canada; published: October 1, 2020).

Bromantane (adamantylbromphenylamine) is an anxiolytic psychostimulant developed and used in Russia at doses of 50-100 $\mathrm{mg}$ for what used to be called neurasthenia. It also is a banned doping agent. ${ }^{1}$ Its scientific literature, found almost exclusively in Russian journals, refers to it an "actoprotectant," that is, a compound that improves physical performance without increasing oxygen consumption or heat production. ${ }^{2}$ Its pharmacology is complex, dose dependent, and insufficiently characterized; dopaminergic, cholinergic, and adrenergic neurotransmission are affected. ${ }^{3}$ In addition, bromantane seems to increase adaption to hypoxia, ${ }^{4}$ which is suggestive of a downregulation of hypoxia-inducible factor 1-alpha (HIF$1 \alpha$ ). It is the last-mentioned feature that prompted the inventors to investigate bromantane in mouse models of fibrotic complications of nonalcoholic fatty liver disease (NAFLD), kidney injury, and lung diseases: all these conditions have HIF- $1 \alpha$ involvement. ${ }^{5-7}$ The results presented in this document are quite convincing, but translational success in the preventive setting will strongly depend on the required human doses.

\section{A NONRESORBABLE ANTIBIOTIC PREVENTS SICKLE CELL VASO-OCCLUSIVE CRISES}

International Patent Application WO/2020/198136, Use of Rifaximin on Circulating Aged Neutrophils in Sickle Cell Disease (Inventor: Lim SH; applicant: New York Medical College, Valhalla, New York, USA; published: October 1, 2020).

Activated and circulating aged neutrophils (CANs) are pivotal for the pathogenesis of vaso-occlusive crises in sickle cell disease: they adhere to vascular endothelium and form the nidus for sickled red blood cells to aggregate on. In mice neutrophil aging is modulated by the intestinal microbiome, ${ }^{8,9}$ which probably is a runaway consequence of the intestinal injury and increased gut permeability caused by the recurrent hypoxia-reperfusion injury induced by vasoocclusive crises. ${ }^{10}$ In these earlier experiments, treatment of sickle cell disease (SCD) mice with an oral cocktail of ampicillin, neomycin, metronidazole, and vancomycin had induced a reduction in CAN counts and protection from fatal vaso-occlusive crises, but absence of a systemic effect of these antibiotics had not been demonstrated. By using rifaximin, a minimally absorbed oral antibiotic for intestinal infections, it has now been shown that the controlling effect on CANs is indeed owing to antibiotic modulation of the intestinal microbiome. Long-term use of rifaximin is an established treatment and does not increase the risk for the development of Clostridium difficile infection, against which SCD patients enjoy a degree of protection.-For the peer review companion paper see Lim et al. ${ }^{11}$

\section{A FREE RADICAL SCAVENGER FOR FATTY LIVER DISEASE} International Patent Application WO/2020/199650, Application of Edaravone in the Treatment or Prevention of Nonalcoholic Steatohepatitis (Inventors: Ding H, Zhou Y; applicant: Suzhou Auzone Biological Technology Co., Ltd, Suzhou, Jiangsu, China; published: October 8, 2020).

Edaravone is a free-radical scavenger that was developed by Mitsubishi Tanabe Corp. It received Japanese approval as a once-monthly intravenous infusion treatment for amyotrophic lateral sclerosis in 2015 and by the U.S. Food and Drug Administration (US FDA) (as Radicava ${ }^{\circledR}$ ) in 2017; the European marketing authorization application was withdrawn in 2019. To date it remains the only agent of this type with explicit treatment approvals for a specific disease. ${ }^{12}$ Based on the ubiquitous involvement of free radicals it has also been investigated (so far without convincing results) in other neurological diseases ${ }^{13}$ and in many diseases associated with ischemia-reperfusion injury. ${ }^{14}$ A Chinese company has now added nonalcoholic fatty liver disease (NAFLD) and steatohepatitis (NASH) to this catalog. This is not surprising given that considerable clinical data have been amassed for edaravone in hepatic diseases, and the fact that its pharmacokinetics are almost unaffected even by severe hepatic 
impairment is an additional bonus. ${ }^{15}$ Surprisingly, although the role of oxidative stress and free radicals in NAFLD and NASH is well known, ${ }^{16}$ there seem to be no reports of studies with edaravone. The inventors compared it (at oral doses of $90 \mathrm{mg} /$ $\mathrm{kg}$ ) with Gilead's candidate Ask1 inhibitor selonsertib (now presumably discontinued) in the commonly used rabbit model of NASH; edaravone was superior in reducing NASH scores (the sum of the scores of steatosis, inflammation, and cell ballooning), elevated liver enzymes, and histopathology.

\section{ASTHMA DRUGS TREAT ANEURYSMS}

\section{AND RELATED VASCULAR DISEASES}

International Patent Application WO/2020/207165, Compound for Treating Vascular Malformation (Inventors: Shan $\mathrm{H}$, Pang P, Mao J, Hu X, He H, Xiao F; applicant: The Fifth Affiliated Hospital of Sun Yat-Sen University, Zhuhai, Guangdong, China; published: October 15, 2020).

Aneurysms are routinely treated by surgery, but research into pharmacological treatments has accelerated during recent years: somatic gene therapies, epigenetic therapies (WO/2019/046791), but also small molecule agents (e.g., tyrosine hydroxylase inhibitors, WO/2019/243653; or S1P receptor agonists, WO/2014/ 175287) have appeared in the patent literature. The genetics of aneurysm propensity are also under intense study ${ }^{17}$ However, the spectrum of vascular malformations extends beyond aneurysms. In earlier work the inventors examined four generations of a family suffering from what they named multiorgan venous and lymphatic defect (MOVLD) syndrome. Using cell culture models and zebrafish knockouts they determined that single nucleotide polymorphism mutations in $D D X 24$, a gene coding for a DEAD box helicase, are responsible not only for the MOVLD syndrome but could also be found in some sporadic cases of vascular malformation, and that DDX24 is involved in early vascular development. ${ }^{18}$ In this patent document the inventors proceed to identify antagonists of the cysteinyl leukotriene receptor-1 as agents to treat or prevent such malformations. These are the asthma drugs based on the "lukast" class of agents (motelukast, zafirlukast, and pranlukast) but also the older necrodomil. See the directly competitive work from the Karolinska Institute, prepublished almost 7 months before the April 2019 priority date of this patent application. ${ }^{19}$

\section{TANDOSPIRONE FOR L-DOPA INDUCED DYSKINESIA:} A MATTER OF ADMINISTRATION

International Patent Application WO/2020/218487, Therapeutic Drug for Dyskinesia (Inventors: Kurita M, Ikeda Y, Nakato M; applicant: Sumitomo Dainippon Pharma Co., Ltd., Osaka, Japan; published: October 29, 2020).
Even three decades after the launch of the first selective dopamine receptor agonists for the treatment of Parkinson's disease, L-dopa remains a mainstay of therapy, although $30 \%-50 \%$ of patients develop dyskinesia within 5 years. Articles reporting and discussing $5-\mathrm{HT}_{1 \mathrm{~A}}$ agonists, which originally carried high hopes for the treatment of anxiety and depression, as treatments for dyskinesia have been published for almost two decades, ${ }^{20}$ also for tandospirone, which is the subject of this patent application. ${ }^{21}$ The inventors apparently believe that patenting is still possible with transdermal administration of tandospirone, based on the different pharmacokinetics and release kinetics, given that L-dopa is orally administered. This is supported by data from a 6-OHDA lesioned rat model of L-dopa dyskinesia. The effect of the tandospirone metabolite, 1-(2-pyrimidyl)piperazine (which is not generated with transdermal administration) was also evaluated, and revealed potential for rebound dyskinesia with oral dosing.

\section{AN ALLERGIC RHINITIS DRUG FOR DEMENTIA}

International Patent Application WO/2020/222799, A Method of Treating Mental, Behavioral, Cognitive Disorders (Inventors: Wang J, Cui G; applicant: La Pharma Tech, Inc., Blacksburg, Virginia, USA; published: November 5, 2020).

The anti-allergic agent, azelastine, has a wider range of pharmacologic effects on mediators of inflammation (including leukotrienes, kinins, platelet-activating factor, tumor necrosis factor alpha [TNF- $\alpha$ ], and inflammatory cell migration) than other histamine $\mathrm{H}_{1}$ receptor antagonists and histamine release blockers of its class. ${ }^{22,23}$ Thirteen years ago a Japanese group published results from a blinded 4-week trial with $2 \times 1 \mathrm{mg}$ azelastine in 30 patients with mild-to-severe dementia ${ }^{24}$; behavioral and psychological symptoms improved but cognition did not. The inventors (and the examiner) do not cite this article but in any case this line of investigation has now been followed up using an unusually high dose of azelastine (12 mg once daily) for 6 months in two elderly patients with advanced dementia. Measurable improvements set in after 6 weeks. If supported by controlled trials this would be remarkable because no anti-inflammatory drug has yet shown efficacy in prospective dementia trials. Azelastine is almost devoid of anticholinergic effects ${ }^{25}$ that would mask or overcompensate beneficial behavioral effects of other antiallergic medications. If the high doses reported here are really required, it would rule out the intranasal route used in azelastine preparations for allergic rhinitis. 


\section{A DRUG FOR NAUSEA AND VOMITING ALSO TREATS MALARIA}

International Patent Application WO/2020/225283, NK1 Inhibitors for the Treatment of Malaria (Inventor: SalinasMarin MV; applicant: Plus Vitech, S. L., Sevilla, Spain; published: November 12, 2020).

This patent application is yet another example of an inadvertent discovery of a repurposing opportunity. An immunosuppressed patient with chemotherapy-refractory lymphoma was given the antiemetic NK1 receptor blocker, aprepitant as an off-label compassionate treatment based on available evidence. ${ }^{26,27}$ Initial doses of 250/160/160 mg t.d.s. were increased to $935 / 935 / 980 \mathrm{mg}$ t.d.s. without adverse events except fatigue and heartburn. Shortly after treatment commenced, the patient was identified as also suffering from malaria. Standard antimalarial drugs triggered severe adverse reactions, and had to be discontinued on day 6. However, treatment with aprepitant was continued and, surprisingly, also resulted in an effective and nontoxic treatment of the patient's malaria after 3 weeks. The inventors speculate that aprepitant either directly causes extensive lysis of the Plasmodium parasite, or by activating the immune system, or a combination of both. This is supported by in vitro experiments with cultures from several chloroquine-resistant Plasmodium falciparum strains exposed to aprepitant concentrations between 5 and $50 \mu \mathrm{M}$, and by successful parasite count reduction in Plasmodium berghei-infected mice treated with the prodrug, fosaprepitant $(2.5-40 \mathrm{mg} / \mathrm{kg}$ for 7 days). Maropitant was also effective. For both drugs, intermediate doses worked best, achieving $>75 \%$ parasite count suppression.-The literature has no reference for NK1 antagonists in malaria, but aprepitant has been reported to show effects in other diseases caused by protozoan parasites, such as cryptosporidiosis $^{28}$ and trypanosomiasis. ${ }^{29}$

\section{A PEPTIDE USED FOR CELIAC DISEASE COULD RESTORE SALIVA AND TEAR FLOW}

International Patent Application WO/2020/227341, Compositions and Methods for Treating Sjögren Syndrome (Inventors: Madan JP, Sivils KL, Deshmukh U, Radhakrishnan B, Woodard K, Bagavant H; applicants: 9 Meters Biopharma, Inc., Raleigh, North Carolina, USA; Oklahoma Medical Research Foundations, Oklahoma City, Oklahoma, USA; published: November 12, 2020).

In Sjögren syndrome lymphocyte infiltration and autoantibodies damage ductal and acinar epithelial cells in exocrine glands, resulting in their dysfunction that causes loss of saliva and tear production, along with other symptoms including leaky gut syndrome. ${ }^{30}$ It is no surprise that these last-mentioned intestinal Sjögren symptoms can be treated with larazotide, an octapeptide that protects tight junctions by blocking zonulin receptors, ${ }^{31}$ and can therefore restore intestinal impermeability in celiac disease. ${ }^{32}$ Combination with aminosalicylic acids, commonly used in intestinal inflammatory diseases, is also obvious. However, treatment of the more classical symptoms-dry eye and dry mouthreflects a higher degree of innovation and has not been reported, although larazotide has been investigated in many inflammatory conditions. ${ }^{33}$ An in vitro assay system to measure tight junction integrity was established using PARC10 cells (an immortalized rat acinar epithelial cell line), which mimics the biochemical and functional characteristics of primary salivary gland acinar cells. Monolayers were incubated for $24 \mathrm{~h}$ with $3 \mathrm{ng} / \mathrm{mL}$ of interferon gamma and TNF- $\alpha$ to cause tight junction disruption. Larazotide acetate ( 1 and $5 \mathrm{mM}$ ) or any one of 4-aminosalicylic acid (4-ASA), 4-amino-phenyl-acetic acid (4-APAA), and 4-acetyl aminophenyl-acetic acid (4-Ac APAA) (5 or $10 \mathrm{mM}$ ) was added to the apical surface and the cells were allowed to grow for an additional $24 \mathrm{~h}$, which restored cytokine-disrupted transepithelial electrical resistance to varying significant degrees. Such concentrations are achievable with eyedrops and in the oral cavity.

\section{AN ANTIDEPRESSANT FOR YET ANOTHER TYPE OF HEADACHE}

International Patent Application WO/2020/229388, Mirtazapine for Use in Medication Overuse Headache Based on Tension-Type Headache (Inventors: Pause A, Bedtsen L, Jensen R, Olesen J; applicants: Curatis AG, Liestal, Switzerland; Rigshospitalet-Glostrup, Glostrup, Denmark; The University of Copenhagen, Copenhagen, Denmark; published: November 19, 2020).

When mirtazapine, a tetracyclic piperazine-azepine antidepressant, was launched in the 1990s it was marketed as a noradrenergic and specific serotonergic antidepressant (NaSSA) to distinguish it from the dominant selective serotonin reuptake inhibitors (SSRIs). Actually its pharmacological activity spectrum is almost as broad as that of the tricyclic psychotropic drugs; it antagonizes $\alpha$-adrenergic, serotonin 5- $\mathrm{HT}_{2}$, and histamine $\mathrm{H}_{1}$ receptors but does not inhibit neurotransmitter reuptake. ${ }^{34}$ In long-term treatment it induces tonic activation of postsynaptic 5-HT receptors. ${ }^{35}$ As most non-SSRI antidepressants mirtazapine is also an analgesic and there have been many reports of its use in various types of 
headache; it is considered a second-choice drug in the most common form, tension-type headache. ${ }^{36}$ There is no great surprise in the fact claimed here, namely its efficacy in medication overuse headache-which is defined as headache occurring on $>15$ days per month in patients with a primary headache disorder (frequently migraine) who regularly overuse one or more headache medications for more than 3 months. ${ }^{37}$ According to the invention there is no need to discontinue the overused drugs before initializing mirtazapine. Data from two patients given 15-30 mg mirtazapine per day for 8 weeks show headache reduction scores and intake of overused medication reduced by up to $80 \%$.

\section{AN ANTIBIOTIC AS A TREATMENT FOR A RARE GENETIC DISEASE}

International Patent Application WO/2020/234496, Moxifloxacin for Use in the Treatment of Spinal Muscular Atrophy (Inventors: Artero Allepuz R, Konieczny PT, López Castel A, Pérez Alonso M; applicants: Universitat de València, Valencia, Spain; Fundación Incliva, Valencia, Spain; published: November 26, 2020).

Almost all spinal muscular atrophy (SMA) patients have a deletion, mutation, or other type of homozygous change in the SMN1 gene, but all nevertheless carry one or more copies of the functional SMN2 gene, whose coding region differs from SMN1 by only one C-to-T nucleotide change in an exon 7 splicing enhancer region. ${ }^{38,39}$ However, SMN2 can compensate for SMN1 loss only to a minimal degree because this change causes predominant skipping of its exon 7 using two separate mechanisms. This is what moxifloxacin, a commonly used fluoroquinolone antibiotic, is supposed to correct according to the present invention-at a tiny fraction of the cost of the antisense oligonucleotide, nursinersen $\left(\right.$ Spinraza ${ }^{\circledR}$ ). Quantification of Smn protein levels in fibroblasts derived from patients with SMA, treated with moxifloxacin at $500 \mu \mathrm{M}$ (the maximum nontoxic concentration) for $72 \mathrm{~h}$, showed an 70\% increase. Moxifloxacin was also successfully evaluated in HeLa cells to determine the existence of Smn aggregates colocalized with Cajal's bodies, which would indicate the presence of functional protein. Treatment with moxifloxacin also allows recovery of fusion capacity in SMA patient myoblasts to levels similar to that of healthy myoblasts.-In their peer review companion article, ${ }^{40}$ the inventor described the screening of 1,100 drugs from the Prestwick Chemical Library in an in vivo SMN2 minigene reporter system in Drosophila motor neurons. SMA is considered a disease for which drug repositioning has particular potential. ${ }^{41}$
FROM RAVE DRUG TO TREATMENT

\section{FOR AN ULTRA-RARE GENETIC DISORDER}

International Patent Application WO/2020/247615, Ketamine and Ketamine/Nap for Treatment of ADNP Syndrome and Related Neurological Conditions (Inventors and applicants: Sermone S, Davis MC, USA; published: December 12, 2020).

Activity-dependent neuroprotective protein syndrome (Helsmoortel-Van der Aa syndrome) is an ultra-rare genetic condition in the spectrum of autism with intellectual disability, caused by loss-of function mutations in the ADNP gene. ${ }^{42}$ These trigger two distinct episignatures, ${ }^{43}$ which correlate only modestly with the clinical phenotype. The Activity-dependent neuroprotective protein (ADNP) protein contains an NAP motif (NAPVSIPQ) that binds to microtubules, which seems to be the structural basis for its pivotal role in neurodevelopment. ${ }^{44}$ The N-methyl-D-aspartate receptor blocker, ketamine increased levels of the pro-apoptotic enzyme-activated caspase-3 (Ac3) in the somatosensory cortex of neonatal mice, an effect that could be reversed by the NAP peptide. ${ }^{45}$ On the contrary, sub-anesthetic doses of ketamine induce ADNP expression and are neuroprotective in a similar paradigm. ${ }^{46}$ The inventors cite both articles (published 2012 and 2015, respectively) but argue that the mice and rats used in these studies did not carry an ADNP mutation, and that claims for treating ADNP syndrome with low-dose ketamine are therefore novel.-Both inventors have offspring afflicted with ADNP syndrome. Sandra Sermone is President and Executive Director at ADNPkids Research Foundation; Matt Davis is a neurologist.

\section{THYROXIN FOR AN INNER EAR DISORDER}

International Patent Application WO/2020/254587, Thyroid Hormone or Metabolite for Use in the Treatment of a Peripheral Vestibulopathy (Inventors: Tighilet B, Chabbert C, Rastoldo G, Pericat D; applicants: Centre National de la Recherche Scientifique, Paris, France; Université d'Aix-Marseille, Marseille, France; published: December 24, 2020).

There are many types of vestibular disorders that are characterized by gait instability, head motion intolerance, and nystagmus. ${ }^{47}$ If these symptoms occur without a demonstrable central lesion and no non-age-related auditory deficit the condition is usually labeled peripheral vestibulopathy, and ascribed to a deafferented vestibular environment. ${ }^{48}$ After the acute manifestation compensatory mechanisms set in, aiming at neurogenesis ${ }^{49}$ and at modulation of excitability of the remaining functional afferents to restore the electrophysiological balance between the homologous left and right 
vestibular nuclei. The inventors observed that after a left-side vestibular neurectomy in rats, treatment with thyroxin (T4) at a dose of $10 \mu \mathrm{g} / \mathrm{kg}$ administered just after lesioning and at days 1,2 , and 3 significantly reduced the vestibular deficits, including the general behavioral alterations during the acute phase, accompanied by improvement of walking and speed motion over several days. The inventors, who have published extensively on animal models of vestibular dysfunction, do not disclose what prompted them to test thyroid hormone in this application, but their reasoning is not difficult to retrace: there is a clear relationship between thyroid autoimmunity (which lowers T4 levels) and vestibular disorders. ${ }^{50}$

\section{A CANKER SORE DRUG FOR HEPATITIS}

International Patent Application WO/2020/259706, Use of Amlexanox in Preparing Anti-hepatitis Virus Drug (Inventors: Wang X, Yang J, Xu X, LI L, Zhao C, Wang Y, et al.; applicant: Marine Biomedical Research Institute of Quingdao Co., Ltd., Qingdao, Shandong, China; published: December 30, 2020).

Amlexanox is an anti-inflammatory immune modulator in widespread use for aphthous ulcers, onto which it is applied as an adhesive paste. In Japan, it is used topically and systemically for a variety of allergic conditions. It seems to have multiple mechanisms among which inhibition of Tbk 1 and IKKe (which activate the innate immune response via the interferon response regulator Irf3) are prominent. The pivotal involvement of these kinases in many diseases has caused amlexanox to be investigated in various cancers, ${ }^{51}$ acute lung and liver injury, ${ }^{52,53}$ myocardial infarction, ${ }^{54}$ multiple sclerosis, ${ }^{55}$ and other conditions. So far viral infections have not been reported as a possible application. The inventors show that amlexanox inhibits hepatitis B virus (HBV) replication in the HepG2 hepatocyte cell line (at $10 \mu \mathrm{M}$ ) and in mouse models (at $50 \mathrm{mg} / \mathrm{kg} /$ day) with an efficacy comparable with that of lamivudine and tenofovir. This is surprising because hepatitis viruses (and other viruses, including dengue and vesicular stomatitis virus) also suppress the Tbk1/IKK $\varepsilon /$ Irf3 axis to evade interferon induction. ${ }^{56}$

\section{REFERENCES}

1. Burnat $P$, Payen $A$, Le Brumant-Payen $C$, Hugon M, Ceppa F: Bromontan, a new doping agent. Lancet 1997;350:963-964.

2. Oliynyk S, Oh S: The pharmacology of actoprotectors: practical application for improvement of mental and physical performance. Biomol Ther (Seoul) 2012; 20:446-456.

3. Sergeeva SA, Krapivin SV, Losev AS, Morozov IS: [Correlated interconnection between pharmacokinetic and dynamic development of the pharmacologic effects of bromantane]. Biull Eksp Biol Med 1995;119:305-308.
4. Morozov IS, Klimova NV, Sergeeva SA, et al.: [Adamantane derivatives enhancing body's resistance to emergencies]. Vestn Ross Akad Med Nauk 1999;3:28-32.

5. Ju C, Colgan SP, Eltzschig HK: Hypoxia-inducible factors as molecular targets for liver diseases. J Mol Med (Berl) 2016;94:613-627.

6. Shu $S$, Wang $Y$, Zheng $M$, et al:: Hypoxia and hypoxia-inducible factors in kidney injury and repair. Cells 2019;8:207.

7. Voelkel NF, Mizuno S, Bogaard HJ: The role of hypoxia in pulmonary vascular diseases: a perspective. Am J Physiol Lung Cell Mol Physiol 2013;304:L457L465.

8. Zhang D, Chen G, Manwani D, et al:: Neutrophil ageing is regulated by the microbiome. Nature 2015;525:528-532.

9. Lim SH, Morris A, Li K, et al:: Intestinal microbiome analysis revealed dysbiosis in sickle cell disease. Am J Hematol 2018;93:E91-E93.

10. Dutta D, Methe B, Amar S, Morris A, Lim SH: Intestinal injury and gut permeability in sickle cell disease. J Trans/ Med 2019;17:183.

11. Lim SH, Dutta D, Moore J: Rifaximin for sickle cell disease. Am J Hematol 2019; 94:E325-E328

12. Writing Group; Edaravone (MCl-186) ALS 19 Study Group. Safety and efficacy of edaravone in well defined patients with amyotrophic lateral sclerosis: a randomised, double-blind, placebo-controlled trial. Lancet Neurol 2017;16:505-512.

13. Watanabe T, Tanaka M, Watanabe K, Takamatsu Y, Tobe A: [Research and development of the free radical scavenger edaravone as a neuroprotectant]. Yakugaku Zasshi 2004;124:99-111.

14. Kikuchi $\mathrm{K}$, Uchikado $\mathrm{H}$, Miyagi $\mathrm{N}$, et al.: Beyond neurological disease: new targets for edaravone (Review). Int J Mol Med 2011;28:899-906.

15. Nakamaru $Y$, Kakubari $M$, Yoshida $K_{1}$ et al:: Open-label, single-dose studies of the pharmacokinetics of edaravone in subjects with mild, moderate, or severe hepatic impairment compared to subjects with normal hepatic functioning. Clin Ther 2020;42:1467-1482.e4.

16. Sumida $Y$, Niki E, Naito $Y$, Yoshikawa $T$ : Involvement of free radicals and oxidative stress in NAFLD/NASH. Free Radic Res 2013;47:869-880.

17. Thakker PD, Braverman AC: Cardiogenetics: genetic testing in the diagnosis and management of patients with aortic disease. Heart 2020 [Epub ahead of print]; DOI: 10.1136/heartjnl-2020-317036.

18. Pang $P, H u X$, Zhou $B$, et al:: DDX24 mutations associated with malformations of major vessels to the viscera. Hepatology 2019;69:803-816.

19. Araújo AC, Tang $X$, Haeggström JZ: Targeting cysteinyl-leukotrienes in abdominal aortic aneurysm. Prostaglandins Other Lipid Mediat 2018;139:24-28.

20. Nicholson SL, Brotchie JM: 5-hydroxytryptamine (5-HT, serotonin) and Parkinson's disease-opportunities for novel therapeutics to reduce the problems of levodopa therapy. Eur J Neurol 2002;9 Suppl 3:1-6.

21. Kannari $K$, Kurahashi $K$, Tomiyama $M$, et al:: Tandospirone citrate, a selective 5-HT1A agonist, alleviates L-DOPA-induced dyskinesia in patients with Parkinson's disease. No To Shinkei 2002;54:133-137.

22. Bernstein JA: Azelastine hydrochloride: a review of pharmacology, pharmacokinetics, clinical efficacy and tolerability. Curr Med Res Opin 2007; 23:2441-2452

23. McTavish D, Sorkin EM: Azelastine. A review of its pharmacodynamic and pharmacokinetic properties, and therapeutic potential. Drugs 1989;38:778-800.

24. Hatakeyama A, Fujii M, Hatakeyama R, Fukuoka Y, Satoh-Nakagawa T, Sasaki H: Azelastine hydrochloride on behavioral and psychological symptoms and activities of daily living in dementia patients. Geriatr Gerontol Int 2008;8:59-61.

25. Rafferty P, Harrison PJ, Aurich R, Holgate ST: The in vivo potency and selectivity of azelastine as an $\mathrm{H} 1$ histamine-receptor antagonist in human airways and skin. J Allergy Clin Immunol 1988;82:1113-1118.

26. Muñoz $M$, Coveñas $R$ : The neurokinin-1 receptor antagonist aprepitant: an intelligent bullet against cancer? Cancers (Basel) 2020;12:2682.

27. Munoz $M$, Covenas $R$, Esteban $F$, Redondo MJ: The substance P/NK-1 receptor system: NK-1 receptor antagonists as anti-cancer drugs. J Biosci 2015;40:441-463.

28. Robinson P, Martin P, Jr., Garza A, D'Souza M, Mastrangelo MA, Tweardy D: Substance $P$ receptor antagonism for treatment of cryptosporidiosis in immunosuppressed mice. J Parasitol 2008;94:1150-1154. 
29. Rodgers J, Bradley B, Kennedy PG: Combination chemotherapy with a substance $P$ receptor antagonist (aprepitant) and melarsoprol in a mouse model of human African trypanosomiasis. Parasitol Int 2007;56:321-324.

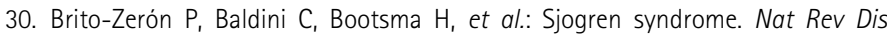
Primers 2016;2:16047.

31. Gopalakrishnan $S$, Durai $M$, Kitchens $K_{\text {, }}$ et al:: Larazotide acetate regulates epithelial tight junctions in vitro and in vivo. Peptides 2012;35:86-94.

32. Khaleghi $S$, Ju JM, Lamba A, Murray JA: The potential utility of tight junction regulation in celiac disease: focus on larazotide acetate. Therap Adv Gastroenterol 2016;9:37-49.

33. Troisi J, Venutolo G, Terracciano $C_{\text {, }}$ et al:: The therapeutic use of the zonulin inhibitor AT-1001 (Larazotide) for a variety of acute and chronic inflammatory diseases. Curr Med Chem 2021 [Epub ahead of print]; DOI: 10.2174/ 0929867328666210104110053.

34. de Boer T: The pharmacologic profile of mirtazapine. J Clin Psychiatry 1996;57 Suppl 4:19-25.

35. Haddjeri N, Blier $P$, de Montigny $C$ : Noradrenergic modulation of central serotonergic neurotransmission: acute and long-term actions of mirtazapine. Int Clin Psychopharmacol 1995;10 Suppl 4:11-17.

36. Bendtsen L, Evers S, Linde M, Mitsikostas DD, Sandrini G, Schoenen J: EFNS guideline on the treatment of tension-type headache-report of an EFNS task force. Eur J Neurol 2010;17:1318-1325.

37. Wakerley BR: Medication-overuse headache. Pract Neurol 2019;19:399-403.

38. Singh RN, Singh NN: Mechanism of splicing regulation of spinal muscular atrophy genes. Adv Neurobiol 2018;20:31-61.

39. Singh NN, Singh RN: How RNA structure dictates the usage of a critical exon of spinal muscular atrophy gene. Biochim Biophys Acta Gene Regul Mech 2019; 1862:194403.

40. Konieczny $P$, Artero R: Drosophila SMN2 minigene reporter model identifies moxifloxacin as a candidate therapy for SMA. FASEB J 2020;34: 3021-3036.

41. Menduti G, Rasà DM, Stanga $S$, Boido $M$ : Drug screening and drug repositioning as promising therapeutic approaches for spinal muscular atrophy treatment. Front Pharmacol 2020;11:592234.

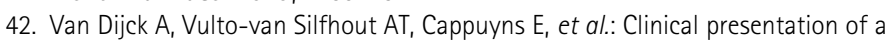
complex neurodevelopmental disorder caused by mutations in ADNP. Biol Psychiatry 2019;85:287-297.

43. Bend EG, Aref-Eshghi E, Everman DB, et al:: Gene domain-specific DNA methylation episignatures highlight distinct molecular entities of ADNP syndrome. Clin Epigenetics 2019;11:64.

44. Malishkevich A, Leyk J, Goldbaum 0, Richter-Landsberg C, Gozes I: ADNP/ ADNP2 expression in oligodendrocytes: implication for myelin-related neurodevelopment. J Mol Neurosci 2015;57:304-313.

45. Turner CP, Gutierrez S, Liu C, et al.: Strategies to defeat ketamine-induced neonatal brain injury. Neuroscience 2012;210:384-392.

46. Brown BP, Kang SC, Gawelek K, et al:: In vivo and in vitro ketamine exposure exhibits a dose-dependent induction of activity-dependent neuroprotective protein in rat neurons. Neuroscience 2015;290:31-40.

47. Strupp $M$, Mandalà $M$, López-Escámez JA: Peripheral vestibular disorders: an update. Curr Opin Neurol 2019;32:165-173.

48. Hegemann SCA, Wenzel A: Diagnosis and treatment of vestibular neuritis/ neuronitis or peripheral vestibulopathy (PVP)? Open questions and possible answers. Otol Neurotol 2017;38:626-631.

49. Tighilet $B$, Chabbert C: Adult neurogenesis promotes balance recovery after vestibular loss. Prog Neurobiol 2019;174:28-35.
50. Chiarella G, Russo D, Monzani F, et al:: Hashimoto thyroiditis and vestibular dysfunction. Endocr Pract 2017;23:863-868.

51. Möller M, Wasel J, Schmetzer J, et al.: The specific IKKepsilon/TBK1 inhibitor amlexanox suppresses human melanoma by the inhibition of autophagy, NFkappaB and MAP kinase pathways. Int J Mol Sci 2020;21:4721.

52. Wang $N$, Li $Y$, Wang $X$, et al:: Inhibition of TBK1 by amlexanox attenuates paraquat-induced acute lung injury. Toxicology 2020;443:152555.

53. Qi J, Zhou Z, Lim CW, Kim JW, Kim B: Amlexanox ameliorates acetaminopheninduced acute liver injury by reducing oxidative stress in mice. Toxicol Appl Pharmacol 2019;385:114767.

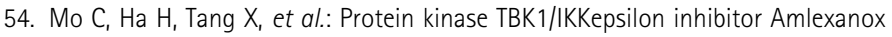
improves cardiac function after acute myocardial infarction in rats. Panminerva Med 2020 [Epub ahead of print]; DOI: 10.23736/S0031-0808.20.03937-3.

55. Quan MY, Song XJ, Liu HJ, et al:: Amlexanox attenuates experimental autoimmune encephalomyelitis by inhibiting dendritic cell maturation and reprogramming effector and regulatory $T$ cell responses. J Neuroinflammation 2019;16:52.

56. Yu S, Chen J, Wu M, Chen $H$, Kato N, Yuan Z: Hepatitis B virus polymerase inhibits RIG-I- and Toll-like receptor 3-mediated beta interferon induction in human hepatocytes through interference with interferon regulatory factor 3 activation and dampening of the interaction between TBK1/IKKepsilon and DDX3. J Gen Virol 2010;91(Pt 8):2080-2090.

Address correspondence to:

Hermann A.M. Mucke, PhD

H.M. Pharma Consultancy

Enenkelstrasse 28/32

Wien A-1160

Austria

E-mail: h.mucke@hmpharmacon.com

\section{Abbreviations Used}

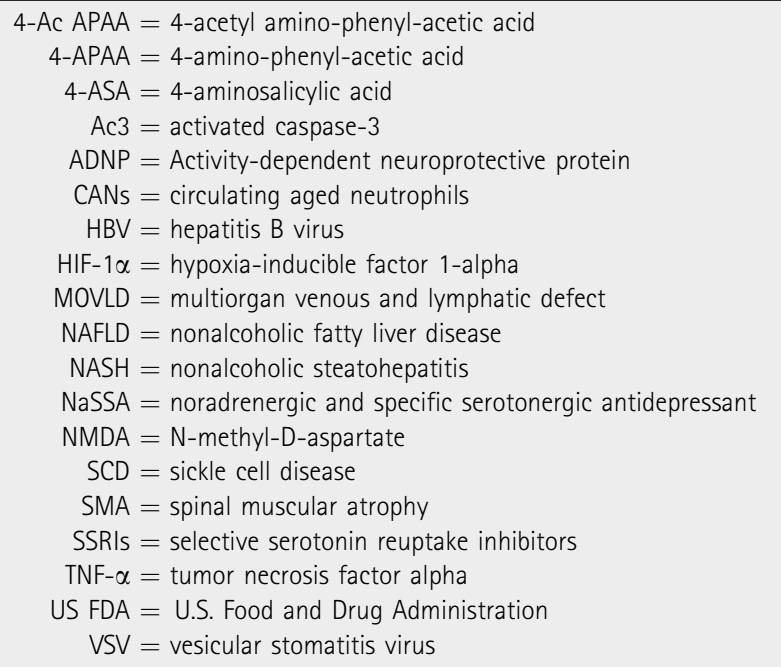

